\title{
Multimodality Treatment for Cardiac Angiosarcoma
}

\author{
Meng Wang ${ }^{1}$, Ganglan $\mathrm{Fu}^{2}$, Huiqi Jiang ${ }^{1}$, Kuan Zeng ${ }^{1}$ and Ping Hua ${ }^{1}$
}

\begin{abstract}
Primary cardiac angiosarcoma is a rare and highly malignant condition. Besides performing complete surgical excision, it remains controversial as to whether survival can be improved with additional treatment. We herein describe a 30-year-old man with a right atrial angiosarcoma. He underwent two operations for the resection of the primary lesion, and the patient's metastatic lesions involved an intestinal segment. With chemotherapy, radiotherapy, and molecular targeted therapy, he survived for 33 months. The literature describing adjuvant therapy for cardiac angiosarcoma, which is mostly case reports, is also reviewed. In conclusion, the limited evidence suggests that multimodality treatment for cardiac angiosarcoma is a beacon of hope to improve the survival of such patients.
\end{abstract}

Key words: cardiac angiosarcoma, chemotherapy, radiotherapy, molecule targeted therapy

(Intern Med 53: 1949-1953, 2014)

(DOI: 10.2169/internalmedicine.53.2470)

\section{Introduction}

Primary cardiac angiosarcoma accounts for around 33\% of all primary malignant cardiac tumors, which make up approximately $25 \%$ of the primary cardiac tumors $(1,2)$. Delayed diagnosis is frequent because the symptoms are not specific, and the incidence of the disease is rare. Once a patient is confirmed to have the disease, the high malignancy of cardiac angiosarcoma is associated with a very poor prognosis. Nonetheless, the therapies for cardiac angiosarcoma remain controversial. Surgical resection has been demonstrated to improve survival (3-6). Researchers have studied the effects of numerous adjuvant therapies, including chemotherapy, radiotherapy, and molecular targeted therapy. Some reports have published negative results for adjuvant therapies that failed to modify the natural course of primary cardiac angiosarcoma. On the other hand, numerous studies have described an increased survival with additional treatments after surgery. However, no randomized controlled trials have been performed to provide evidence that supports or opposes the administration of adjuvant therapies. We herein describe a patient with primary cardiac angiosarcoma who received multimodality adjuvant treatments and survived for 33 months, reinforcing the hope of extending the survival of cardiac angiosarcoma patients with multimodality adjuvant treatments.

\section{Case Report}

A 30-year-old man had fever and chest tightness for more than 40 days. Echocardiography performed in the local hospital showed an ejection fraction (EF) of $45 \%$ and a large pericardial effusion. He was diagnosed with tuberculous pericarditis and received anti-tuberculosis treatment, including rifampin, isoniazid, pyrazinamide, and ethambutol for two weeks. Thereafter the symptoms subsided. Repeat echocardiography showed that the pericardial effusion had been absorbed, and the EF was $73 \%$. However, an abnormal echo signal next to the right atrium was found at this time (Fig. 1), and he was therefore referred to our hospital. Magnetic resonance imaging revealed bilateral pleural effusion, and an abnormal pericardial signal adjacent to the right atrium (Fig. 2). The radiologists considered it to possibly be a mesenchymal tissue tumor. Positron emission tomography scanning confirmed high metabolic activity in the right atrial mass (Fig. 3A) and revealed another high metabolic nodule in the anorectal area. The nodule was resected during a colonoscopy and was confirmed to be an adenoma by pathologists. No other systemic metastases were found.

${ }^{1}$ Department of Cardiac Surgery, Sun Yat-sen Memorial Hospital, Sun Yat-sen University, China and ${ }^{2}$ Department of Anesthesiology, Sun Yat-sen Memorial Hospital, Sun Yat-sen University, China

Received for publication January 11, 2014; Accepted for publication February 20, 2014

Correspondence to Dr. Meng Wang, surgeonwm@outlook.com 


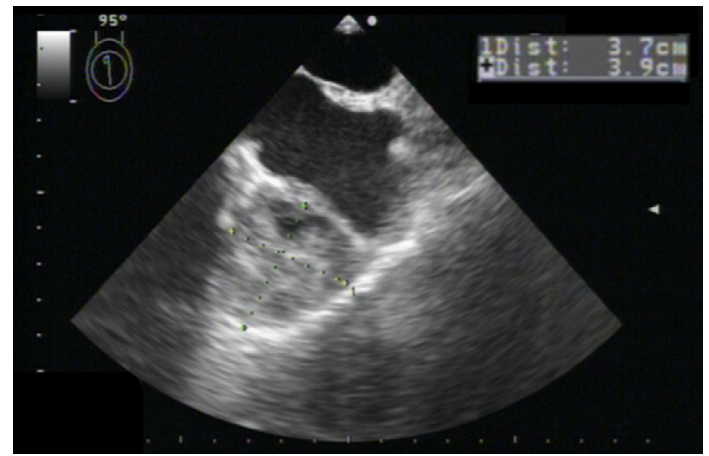

Figure 1. The patient's ultrasound cardiograph showed a pericardial mass measuring approximately $3.7 \times 3.9$ centimeters in size on a two-dimensional display. The mass compressed the right atrium outside of the free wall, which was closely adjacent to and maybe invaded by the mass.
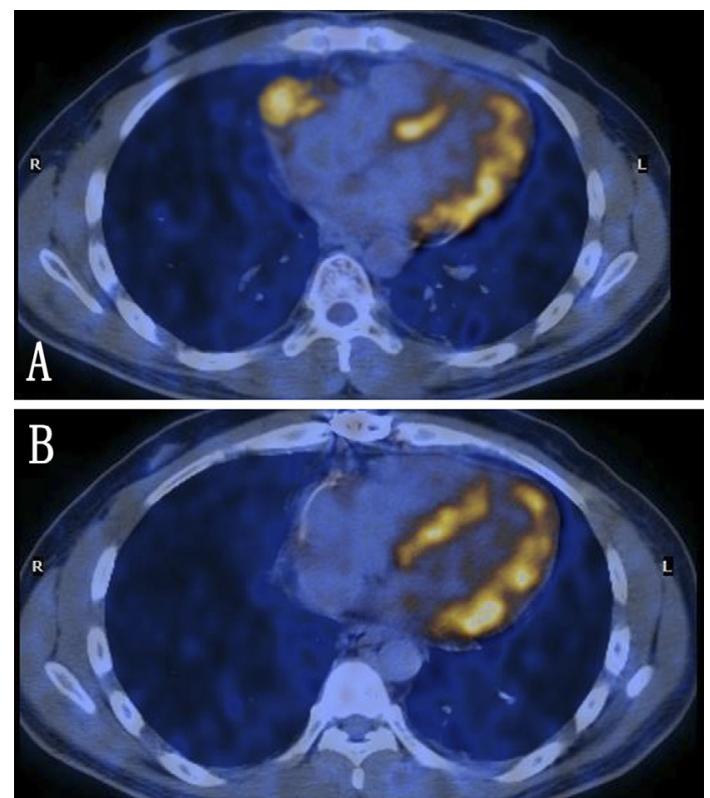

Figure 3. Positron emission tomography confirmed high metabolic activity in the right atrial mass (A). One year after the surgery, positron emission tomography showed the patch used for right atrial reconstruction, and the residual lesion adjacent to the patch showed no evidence of progression (B).

Three months after the first diagnosis, the patient underwent resection of the right atrial mass. He had an extensive adhesion between the pericardium and the right atrium. The mass was located at the anterior inferior wall of the right atrium. The size was approximately $5 \times 4 \times 3 \mathrm{~cm}$. The tumor also invaded the right ventricle adjacent to the right atrial tumor. After cardiopulmonary bypass was established, the tumor was resected along the tricuspid annulus and atrioventricular groove, and a Gortex patch was used to reconstruct the right atrium. The patient's postoperative recovery was uneventful. The pathological report confirmed the diagnosis of a highly differentiated angiosarcoma (Fig. 4A, B), and the margin of the specimen was determined to be tumor tissue. The immunohistochemistry results were as follows:
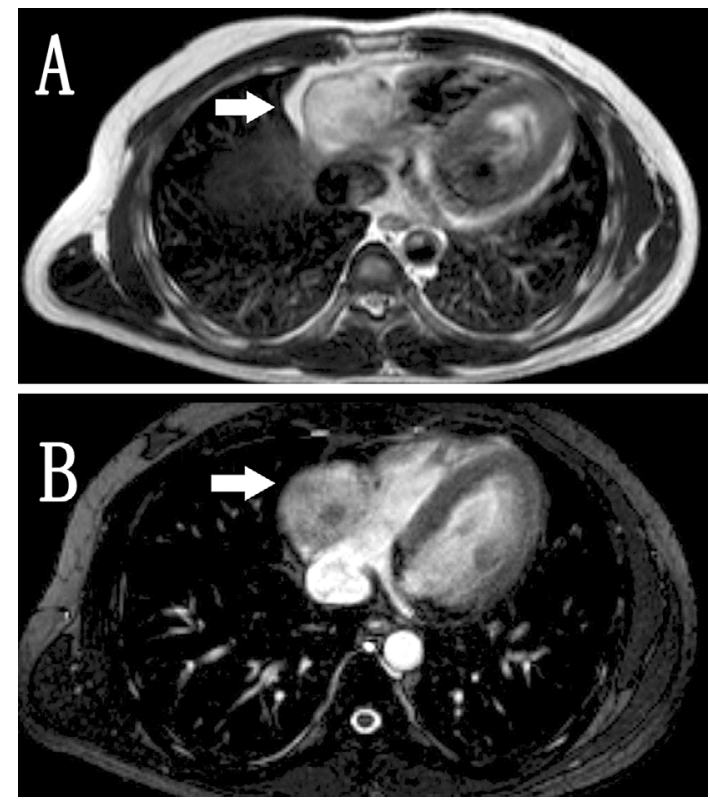

Figure 2. A magnetic resonance imaging scan revealed that the tumor (white arrow) had an isotense to slightly high-intensity signal on a T1-weighted image (A), and an isotense to slightly low-intensity signal on a T2-weighted image (B).
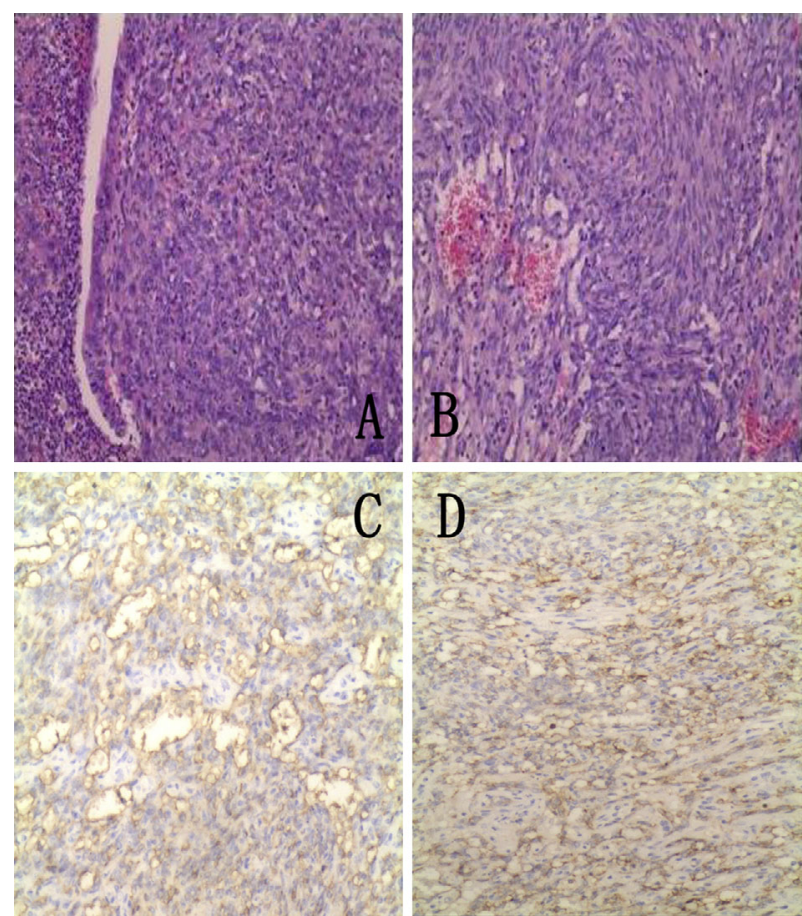

Figure 4. A photomicrograph revealing oval-to-spindle cells with frequent mitoses. There was a vascular neoformation with intraluminal red blood cells (AB). The immunohistochemistry findings were positive for CD31 (C) and F8 (D).

vimentin (+), CD34(+), CD31(+) (Fig. 4C), F8(+) (Fig. 4D), Ki67 40\%(+), actin (-), desmin (+), CD117(-), DOG-1(-), S100(-), and CK(-).

Fourteen days later, he began to receive chemotherapy with docetaxel $120 \mu \mathrm{g}$ once a month. He underwent radical radiotherapy 2 months later. One year after the surgery, in 

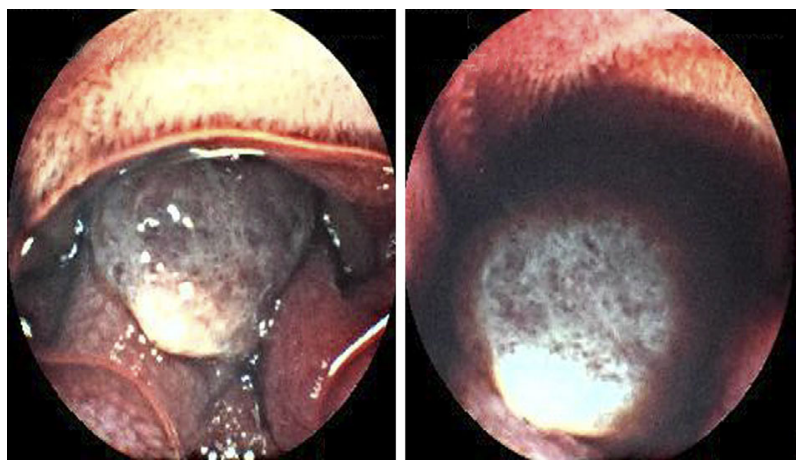

Figure 5. Capsule endoscopy of the jejunum revealed multiple masses with smooth surfaces and focal bleeding.

the 15th month after he was first diagnosed, a follow-up positron emission tomography scan indicated that the residual lesion had not progressed (Fig. 3B).

In the 22nd month, he had manifestations of gastrointestinal bleeding, associated with such symptoms as malena, dizziness, and fatigue. The patient's hemoglobin level decreased to $5.6 \mathrm{~g} / \mathrm{dL}$. However, we found a normal gastrointestinal appearance with gastroendoscopy once and colonoscopy twice. Finally, capsule endoscopy allowed gastroenterologists to confirm multiple metastases in the jejunum (Fig. 5). In the 23rd month, gastrointestinal surgeons resected the metastatic lesions, which were located 80,100, and 140 centimeters distal to the treitz ligament and were $2 \times 1.5,2 \times 2$, and $2 \times 1.5 \mathrm{~cm}$ in size, respectively.

The pathological analysis of the lesions revealed angiosarcoma with transmural invasion (Fig. 6A). Immunohistochemistry showed that the specimens were positive for vimentin, CD34, and CD31 (Fig. 6B). The resected intestinal segment was 80 centimeters long, and the ends were free of tumor tissue. No evidence of any mesenteric lymphnode metastasis was found.

The chemotherapy plan was changed to doxirubicin and ifosfamide. He also received bevacizumab (Avastin) for targeted therapy. However, the primary lesion progressed rapidly and metastasized extensively to the bilateral lungs, pleura, liver, and peritoneum. The patient died of circulatory and respiratory failure 33 months after he first presented at the hospital.

\section{Discussion}

Angiosarcoma is the most common primary cardiac malignant tumor. The incidence is extremely rare, with a postmortem incidence of $0.0001 \%$ (7). The clinical diagnosis of angiosarcoma is often difficult because there are no specific symptoms associated with the disease. Some of the more common cardiac symptoms are chest pain, palpitations, and symptoms of congestive heart failure or acute right heart failure secondary to a pericardial tamponade (8). Systemic symptoms occur in approximately $10 \%$ of cases and include fever, night sweats, and weight loss (9). Metastases occur in approximately $66-89 \%$ of cases at the time of diagnosis and

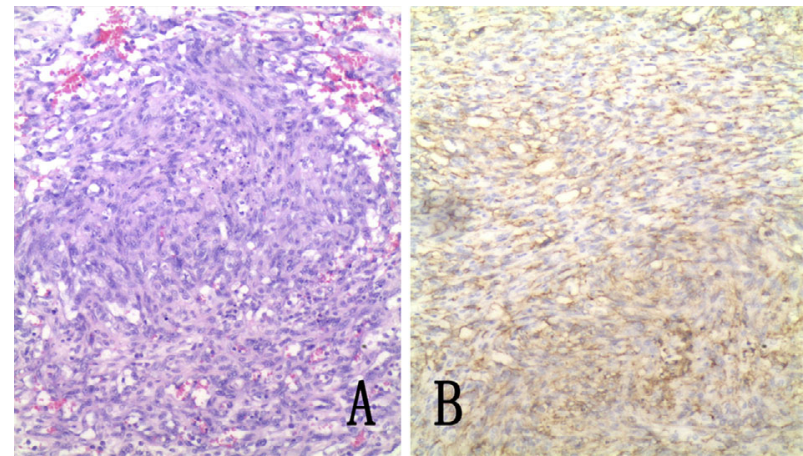

Figure 6. A photomicrograph showing that the metastatic lesion exhibited a spindle cell pattern similar to the primary tumor (A). Immunohistochemistry of the lesion revealed labeling for CD31 (+) (B).

are mostly found in the lungs, liver, bone, lymph nodes, and central nervous system (10). The present patient had fever and pericardial effusion, which was diagnosed as tuberculosis pericarditis despite the absence of night sweats and weight loss. Doctors in the local hospital did not perform pericardiocentesis or administer any other drugs besides the anti-tuberculosis therapy. However, the symptoms remitted, and the patient's effusion decreased after the therapy.

Surgical resection remains the first-line treatment of choice. The applications and benefits of adjuvant therapy, including chemotherapy, radiotherapy, targeted therapy, and others, are debatable.

However, chemotherapy and radiotherapy have wellestablished postoperative roles because of the high probability of metastasis (11). If the tumor is resectable, a multidisciplinary approach with adjunctive chemotherapy and radiotherapy can offer the potential of improved survival $(3,11-25)$ (Table).

With regard to molecular targeted therapy, phase II trials of sorafenib (26), imatinib (27), and bevacizumab (28) for the treatment of advanced or metastatic angiosarcoma have demonstrated the efficacy of these anti-angiogenic drugs. Imatinib was not found to be an active agent against advanced sarcoma. Sorafenib exerts activity against angiosarcoma, with 3.8 and 14.9 months progression-free survival and overall survival, respectively. While sorafenib provides a better progression-free survival, the response rate is lower than those for standard cytotoxic agents. Bevacizumab is effective for angiosarcoma with 57\% (13/23) patients showing a response and no progression.

The longest survival of a patient with cardiac angiosarcoma in the literature was reported by Look et al. in 2012. The patient survived for 84 months following treatment with surgery, chemotherapy, and radiotherapy. The second-longest survivor was described by Kim et al. in 2008; that patient survived for 64 months and also received multidisciplinary treatment.

Our patient could only undergo a palliative debulking resection of the primary lesion. Twenty months later, he underwent a second palliative surgery to excise the metastatic 
Table. Literature Review of Cardiac Angiosarcoma: Treatment Modality and Survival

\begin{tabular}{|c|c|c|c|c|c|}
\hline Treatment & Reference & Published Year & $\mathrm{n}$ & $\begin{array}{l}\text { Age } \\
\text { (Years) }\end{array}$ & $\begin{array}{l}\text { Survival } \\
\text { (Months) }\end{array}$ \\
\hline \multirow{5}{*}{$\begin{array}{l}\text { Surgical resection } \\
\text { alone }\end{array}$} & 3 & 2012 & 1 & 31 & $5+$ \\
\hline & 12 & 2013 & 1 & 80 & 11 \\
\hline & 13 & 2010 & 1 & 41 & 6 \\
\hline & 14 & 2009 & 1 & 56 & 6 \\
\hline & 15 & 2009 & 1 & NR & 0.5 \\
\hline \multirow[t]{5}{*}{ Chemotherapy alone } & 12 & 2013 & 2 & $35-38$ & $7-17$ \\
\hline & 16 & 2012 & 1 & 43 & $12+$ \\
\hline & 16 & 2012 & 1 & 55 & $12-$ \\
\hline & 17 & 2011 & 1 & 60 & $3+$ \\
\hline & 18 & 2007 & 1 & 29 & 3 \\
\hline \multirow{8}{*}{$\begin{array}{l}\text { Surgery plus adjuvant } \\
\text { chemotherapy or } \\
\text { radiotherapy or both }\end{array}$} & 15 & 2009 & 8 & NR & $2-58$ \\
\hline & 16 & 2012 & 3 & $20-45$ & $12+$ \\
\hline & 18 & 2007 & 2 & $31-47$ & $13+-16$ \\
\hline & 19 & 2012 & 11 & $24-53$ & 1-84 \\
\hline & 20 & 2008 & 10 & $20-61$ & $1-64$ \\
\hline & 21 & 1997 & 1 & 8 & $53+$ \\
\hline & 22 & 2005 & 1 & 57 & 48 \\
\hline & 23 & 2012 & 1 & 37 & 32 \\
\hline \multirow{4}{*}{$\begin{array}{l}\text { Chemotherapy plus } \\
\text { molecular targeted } \\
\text { therapy }\end{array}$} & 11 & 2013 & 1 & 25 & $27+$ \\
\hline & 15 & 2009 & 1 & NR & $33+$ \\
\hline & 24 & 2012 & 1 & 31 & $10+$ \\
\hline & 25 & 2006 & 1 & 32 & $6+$ \\
\hline
\end{tabular}

lesion involving the intestinal segment. With the combination of aggressive surgical treatment, chemotherapy, radiotherapy and targeted therapy, the patient survived for 33 months. However, two events should be noted regarding this case. Firstly, the local hospital misdiagnosed the patient with tuberculosis and administered anti-tuberculosis therapy, which did have some effect on improving his symptoms. This did allow us to discover the cardiac mass and estimate the malignancy using imaging technologies without the influence of effusion. Secondly, this is the first report describing intestinal metastasis from cardiac angiosarcoma.

The authors state that they have no Conflict of Interest (COI).

\section{References}

1. Shahani L, Beckmann M, Vallurupalli S. Cardiac angiosarcomaassociated membranoproliferative glomerulonephropathy. Case Rep Med 2011: 956089, 2011.

2. Matheis G, Beyersdorf F. Primary cardiac angiosarcoma. A case report. Cardiology 86: 83-85, 1995.

3. Park WK, Jung SH, Lim JY. Cardiac angiosarcoma on the right atrium: two cases. Korean J Thorac Cardiovasc Surg 45: 120-123, 2012.

4. Penel N, Marreaud S, Robin YM, Hohenberger P. Angiosarcoma: state of the art and perspectives. Crit Rev Oncol Hematol 80: 257263, 2011.

5. McFadden PM, Ochsner JL. Atrial replacement and tricuspid valve reconstruction after angiosarcoma resection. Ann Thorac Surg 64: 1164-1166, 1997.

6. Llombart-Cussac A, Pivot X, Contesso G, et al. Adjuvant chemotherapy for primary cardiac sarcomas: the IGR experience. $\mathrm{Br} \mathrm{J}$ Cancer 78: 1624-1628, 1998.
7. Kodali D, Seetharaman K. Primary cardiac angiosarcoma. Sarcoma 2006: 39130, 2006.

8. Chalhoub E, Mattar BI, Shaheen W, Schulz TK. Cardiac angiosarcoma presenting with tamponade. Intern Med 51: 2905-2907, 2012.

9. Bruce CJ. Cardiac tumours: diagnosis and management. Heart 97: 151-160, 2011.

10. Burke AP, Cowan D, Virmani R. Primary sarcomas of the heart. Cancer 69: 387-395, 1992.

11. Bellitti R, Buonocore M, De Rosa N, Covino FE, Casale B, Sante P. Primary cardiac angiosarcoma in a 25 -year-old man: excision, adjuvant chemotherapy, and multikinase inhibitor therapy. Tex Heart Inst J 40: 186-188, 2013.

12. Fatima J, Duncan AA, Maleszewski JJ, et al. Primary angiosarcoma of the aorta, great vessels, and the heart. J Vasc Surg 57: 756-764, 2013.

13. Puppala S, Hoey ET, Mankad K, Wood AM. Primary cardiac angiosarcoma arising from the interatrial septum: magnetic resonance imaging appearances. Br J Radiol 83: e230-e234, 2010.

14. Yoshitake I, Hata M, Sezai A, et al. Cardiac angiosarcoma with cardiac tamponade diagnosed as a ruptured aneurysm of the sinus valsalva. Jpn J Clin Oncol 39: 612-615, 2009.

15. Bakaeen FG, Jaroszewski DE, Rice DC, et al. Outcomes after surgical resection of cardiac sarcoma in the multimodality treatment era. J Thorac Cardiovasc Surg 137: 1454-1460, 2009.

16. Yusuf SW, Bathina JD, Qureshi S, et al. Cardiac tumors in a tertiary care cancer hospital: clinical features, echocardiographic findings, treatment and outcomes. Heart Int 7: e4, 2012.

17. Baeza-Roman A, Latour-Perez J, Alzamora DR, et al. Prinzmetal's angina in a patient with angiosarcoma of the right cardiac chambers. Heart Lung 40: 346-348, 2011.

18. Mayer $\mathrm{F}$, Aebert $\mathrm{H}$, Rudert $\mathrm{M}$, et al. Primary malignant sarcomas of the heart and great vessels in adult patients--a single-center experience. Oncologist 12: 1134-1142, 2007.

19. Look Hong NJ, Pandalai PK, Hornick JL, et al. Cardiac angiosarcoma management and outcomes: 20-year single-institution expe- 
rience. Ann Surg Oncol 19: 2707-2715, 2012.

20. Kim CH, Dancer JY, Coffey D, et al. Clinicopathologic study of 24 patients with primary cardiac sarcomas: a 10-year single institution experience. Hum Pathol 39: 933-938, 2008.

21. Nakamichi T, Fukuda T, Suzuki T, Kaneko T, Morikawa Y. Primary cardiac angiosarcoma: 53 months' survival after multidisciplinary therapy. Ann Thorac Surg 63: 1160-1161, 1997.

22. Centella T, Oliva E, Garcia Andrade I, Lamas MJ, Epeldegui A. A patient with cardiac angiosarcoma who survived for four years. Case report and literature review. Rev Esp Cardiol 58: 310-312, 2005.

23. Pigott $\mathrm{C}$, Welker M, Khosla P, Higgins RS. Improved outcome with multimodality therapy in primary cardiac angiosarcoma. Nat Clin Pract Oncol 5: 112-115, 2008.

24. Ong P, Greulich S, Schumm J, et al. Favorable course of pericardial angiosarcoma under paclitaxel followed by pazopanib treat- ment documented by cardiovascular magnetic resonance imaging. Circulation 126: e279-e281, 2012.

25. Batzios S, Michalopoulos A, Kaklamanis L, et al. Angiosarcoma of the heart: case report and review of the literature. Anticancer Res 26: 4837-4842, 2006.

26. Maki RG, D'Adamo DR, Keohan ML, et al. Phase II study of sorafenib in patients with metastatic or recurrent sarcomas. J Clin Oncol 27: 3133-3140, 2009.

27. Chugh R, Wathen JK, Maki RG, et al. Phase II multicenter trial of imatinib in 10 histologic subtypes of sarcoma using a bayesian hierarchical statistical model. J Clin Oncol 27: 3148-3153, 2009.

28. Agulnik M, Yarber JL, Okuno SH, et al. An open-label, multicenter, phase II study of bevacizumab for the treatment of angiosarcoma and epithelioid hemangioendotheliomas. Ann Oncol 24: 257-263, 2013.

(C) 2014 The Japanese Society of Internal Medicine http://www.naika.or.jp/imonline/index.html 